Copyright (C) 2013 IEEE. Personal use of this material is permitted. Permission from IEEE must be obtained for all other uses, in any current or future media, including reprinting/republishing this material for advertising or promotional purposes, creating new collective works, for resale or redistribution to servers or lists, or reuse of any copyrighted component of this work in other works. 


\section{Single-Carrier Iterative Frequency-Domain Equalization with Soft Decision Feedback in Shallow Underwater Acoustic Communication}

\author{
Yuanbiao Ou, Zhiqiang He, Weipeng Jiang, \\ Sichuan Guo, and Kai Niu \\ Key Lab of Universal Wireless Communications, \\ Ministry of Education \\ Beijing University of Posts and Telecommunications \\ Beijing, China
}

\author{
Yue Rong*, Michael Caley ${ }^{\dagger}$, \\ and Alec J Duncan ${ }^{\dagger}$ \\ *Department of Electrical and Computer Engineering \\ ${ }^{\dagger}$ Department of Imaging and Applied Physics \\ Curtin University, Bentley, WA, Australia
}

\begin{abstract}
This paper investigates a single-carrier iterative frequency-domain equalization (SC-IFDE) scheme for high-rate underwater acoustic (UA) communication systems. This scheme is based on the minimum mean-squared error (MMSE) criterion, and soft decision feedback is applied to improve the reliability of the equalizer decision. The proposed algorithm is applied to the data received during the UA communication experiment conducted in December 2012 in the Indian Ocean off Rottnest Island, Western Australia. It is demonstrated that using one transmitting transducer and one receiving hydrophone, the proposed SC-IFDE algorithm achieves an average of $3 \%$ uncoded bit-error-rate (BER) with quaternary phase shift keying (QPSK) modulated signals over a range of $1 \mathrm{~km}$.
\end{abstract}

Index Terms-Underwater acoustic communication, iterative frequency-domain equalization, soft decision feedback.

\section{INTRODUCTION}

Acoustic communication in the underwater channel is very challenging because of extremely limited bandwidth, severe fading, strong multipath interference, and significant Doppler shifts [1]. The fading of underwater acoustic (UA) channels exists both as time selective fading with spectral dispersion and frequency selective fading with time dispersion, which is more severe compared with that of terrestrial radio channels [2]. The frequency selective fading in the UA channel is caused by multipath spread, resulting in severe inter-symbol interference (ISI) for high-rate communication [3]. The time selective fading is mainly introduced by the motion of the transmitter, receiver, and the water, leading to rapidly timevarying channel responses.

In December 2012, we conducted an UA communication experiment in the Indian Ocean off Rottnest Island, Western Australia. The main purpose of this experiment was to test the performance of algorithms that we developed to address the following three major challenges in coherent high-rate UA communication system design: synchronization, channel estimation, and channel equalization. In the experiment, there was

This work was supported by the National Science and Technology Major Project of China (No. 2012ZX03004005-002) and the Australian Research Council's Discovery Projects funding scheme (Project Number DP110100736) one transmitting transducer and one receiving hydrophone, and the distance between the transmitter and receiver varied from $125 \mathrm{~m}$ to $10 \mathrm{~km}$. In this paper, we focus on the design of a single-carrier iterative frequency-domain equalization (SCIFDE) algorithm at the receiver and analyzing its performance using the data received during the experiment.

It is shown in [4] that in a dispersive channel environment, SC-FDE has many advantages over single-carrier time-domain equalization (SC-TDE) and orthogonal frequency division multiplexing (OFDM). Firstly, the computational complexity of the SC-FDE is much lower than that of the SC-TDE, especially in high-rate UA communication where the ISI may span over 100 symbols. Secondly, compared with OFDM systems, single carrier systems have a lower peak-to-average power ratio (PAPR). Thus, in our experimental communication system, we use the SC-FDE algorithm to process the UA communication data received from the hydrophone.

The simplest SC-FDE is linear equalization, including zeroforcing (ZF) equalization and MMSE equalization. Compared with ZF equalization, MMSE equalization has a better performance in practical application, since it considers the noise power. However, the performance of linear equalization schemes deteriorates as the ISI and the Doppler effect of the UA channel increase. Nonlinear equalization, such as decision feedback equalization (DFE) with time-domain (TD) or frequency-domain (FD) hard decision feedback was proposed in [5] and [6]. It has been shown that the nonlinear FDE has a better performance than the linear FDE. In [7], an iterative equalization algorithm with TD soft decision feedback and FD soft decision feedback was proposed, which demonstrates an improved performance over linear equalizers. In this work, we use the SC-IFDE with FD soft decision feedback to process the signals received from the hydrophone in our UA communication experiment with the aim of achieving a lower bit-error-rate (BER) than linear MMSE equalization.

The results of our experiment show that the performance of the SC-IFDE is much better than linear MMSE equalization. In particular, the proposed SC-IFDE algorithm achieves an 


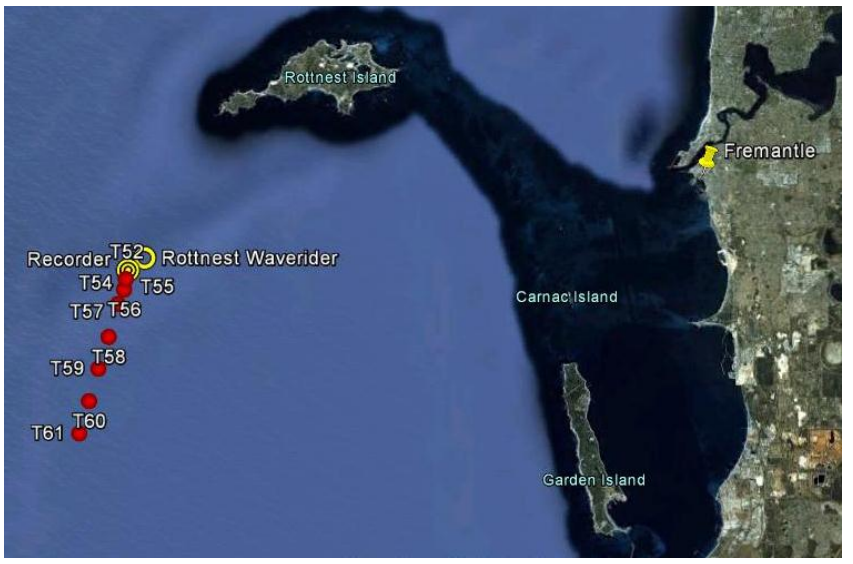

Fig. 1. General location of the experiment environment along $50 \mathrm{~m}$ depth contour

average of 3\% uncoded BER with quaternary phase shift keying (QPSK) modulated signals over a range of $1 \mathrm{~km}$, which is $3 \mathrm{~dB}$ lower than the average BER (about 6\%) obtained by linear MMSE equalization.

\section{EXPERIMENT AND SYSTEM MODEL}

\section{A. Experiment Arrangement}

An UA communication experiment was conducted in December 2012 over distances of $125 \mathrm{~m}$ to $10 \mathrm{~km}$ in the Indian Ocean off Rottnest Island, Western Australia, as shown in Figure 1. The receiver (recorder) was located on the sea bed close to the Rottnest Waverider Buoy. The red dots with labels of T52, T54, T55, T56, T57, T58, T59, T60, and T61 denote the transmitter positions which were $125 \mathrm{~m}, 250 \mathrm{~m}, 500 \mathrm{~m}, 1$ $\mathrm{km}, 2 \mathrm{~km}, 4 \mathrm{~km}, 6 \mathrm{~km}, 8 \mathrm{~km}$, and $10 \mathrm{~km}$ from the receiver, respectively. The average water depth was $50 \mathrm{~m}$.

The transmitter and receiver arrangements are illustrated in Figure 2. For the transmitter, a single transducer was attached to a drifting vessel by a cable, and the nominal transmitter transducer depth was $20 \mathrm{~m}$. A single hydrophone at the receiver was attached through a cable so that the hydrophone was $1 \mathrm{~m}$ above the seabed. A drifting transmitter was selected to give flexibility in exploring different communication ranges. However, such a flexible arrangement allows movement of both transducer and hydrophone, increasing the Doppler shifts and Doppler spreading, thus making channel estimation and tracking more challenging. According to the GPS data, at the $1 \mathrm{~km}$ range, the average drift speed of the vessel was 0.96 $\mathrm{m} / \mathrm{s}$, with a peak drift speed of $1.7 \mathrm{~m} / \mathrm{s}$.

Signals were transmitted at the $12 \mathrm{kHz}$ carrier frequency of the transmit transducer. The system bandwidth was 4 $\mathrm{kHz}$. Transmitted and received signals were sampled with 24 bit resolution at $96 \mathrm{kHz}$. 8PSK and QPSK symbols were transmitted for the range of $125 \mathrm{~m}, 250 \mathrm{~m}, 500 \mathrm{~m}, 1 \mathrm{~km}, 2$ $\mathrm{km}$, and $4 \mathrm{~km}$. QPSK and BPSK symbols were transmitted for the range of $6 \mathrm{~km}$ and $8 \mathrm{~km}$. For the range of $10 \mathrm{~km}$, BPSK symbols were transmitted.

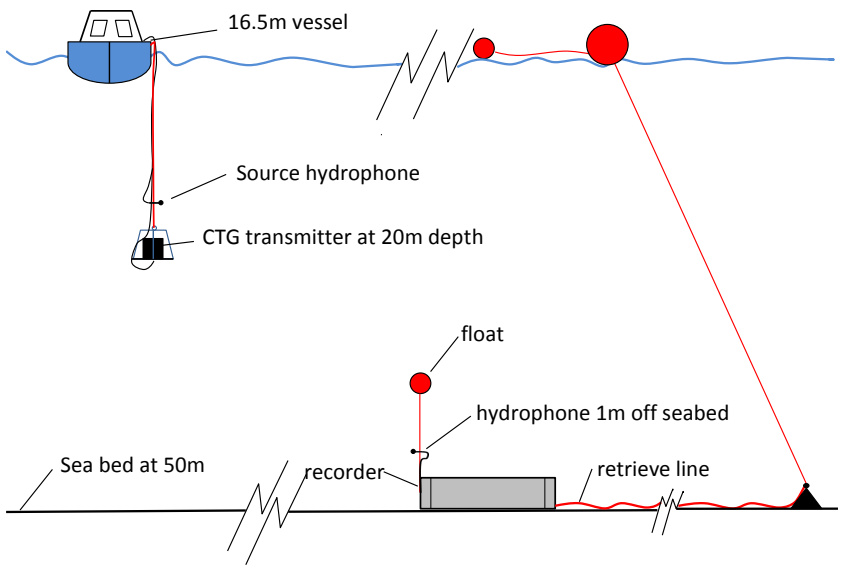

Fig. 2. Transmitter and receiver diagram

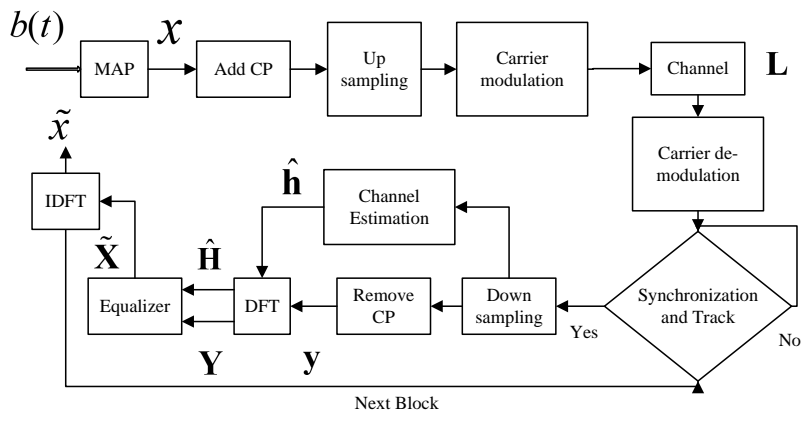

Fig. 3. System model

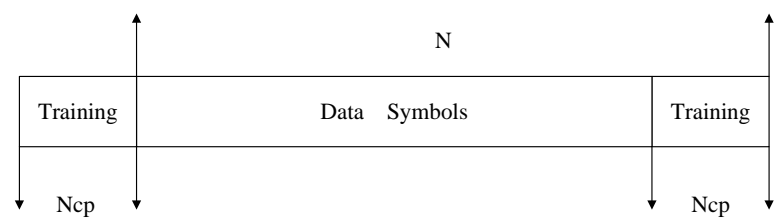

Fig. 4. Block structure with pilot and data symbols

\section{B. System Model}

Figure 3 shows the UA communication system model in our experiment. At the transmitter, vectors of $M$ informationcarrying bits $\mathbf{b}_{k}=[b((k-1) M+1), \ldots, b(k M)]^{T}$, where $b(t) \in\{0,1\}$, are first mapped into symbols $x(k)$ based on the set of normalized constellations $\mathbf{S}=\left\{s_{1}, s_{2}, \ldots, s_{2^{M}}\right\}$. The mapped symbols are then parsed into blocks of $N-N_{c p}$ data symbols with $N_{c p}$ training symbols in the end as $\mathrm{x}=$ $[x(1), \ldots, x(N)]^{T}$. For notational simplicity, we omit the index on the symbol blocks. A block of $N_{c p}$ training symbols is added to the front and the end of each block, as shown in Figure 4 . The training block in the front is also used as the cyclic prefix $(\mathrm{CP})$. 
The equivalent block of baseband received symbols after removing the $\mathrm{CP}$ is given by

$$
y(k)=\sum_{l=0}^{L-1} h(l) x(k-l)+n(k), k=1, \ldots, N
$$

where $h(l), l=0,1, \ldots, L-1$ are the equivalent channel impulse responses (CIRs), including the effect of transmitter pulse shaping filter, the UA channel, and the receiver matched filter, $L$ stands for the order of the underwater multipath channel, and $n(k)$ is the additive noise. We can also express (1) in vector-matrix form as

$$
\mathbf{y}=\mathbf{L x}+\mathbf{n}
$$

where $\mathbf{L}$ is an $N \times N$ circulant matrix whose first column is $\left[h(0), h(1), \ldots, h(L-1), \mathbf{0}_{1 \times(N-L)}\right]^{T}$. We assume that the CIRs are quasi-static within one data block after the Doppler compensation. In our experimental UA communication system, the CIRs are estimated using the compressed sensing (CS) based technique at the receiver.

To convert the time-domain received symbols into the frequency-domain, we apply a normalized discrete Fourier transform (DFT) matrix $\mathbf{F}$ of size $N \times N$ whose $(m, n)$-th element is given by $\frac{1}{\sqrt{N}} \exp \left(\frac{-j 2 \pi(m-1)(n-1)}{N}\right)$. Since $\mathbf{L}$ is a circulant matrix, and $\mathbf{F}$ satisfies $\mathbf{F}^{H} \mathbf{F}=\mathbf{I}_{N}$, where $(\cdot)^{H}$ stands for the matrix Hermitian transpose and $\mathbf{I}_{N}$ is an $N \times N$ identity matrix, the frequency-domain received symbol vector can be written as

$$
\mathbf{Y}=\mathbf{F y}=\mathbf{F L F}^{H} \mathbf{F x}+\mathbf{F n}=\mathbf{H X}+\mathbf{N}
$$

where $\mathbf{H}=\mathbf{F} \mathbf{L} \mathbf{F}^{H}$ is a diagonal matrix whose diagonal elements are the frequency responses of CIRs, $\mathbf{X}=\mathbf{F x}$ and $\mathbf{N}=\mathbf{F n}$ are the frequency domain signal and noise vectors, respectively. The $k$-th diagonal element of $\mathbf{H}$ is given by

$$
H_{k}=\frac{1}{\sqrt{N}} \sum_{l=0}^{L-1} h(l) \exp \left(\frac{-j 2 \pi(k-1) l}{N}\right), k=1, \ldots, N .
$$

\section{ITERATIVE FREQUENCY-DOMAIN EQUALIZATION ALGORITHM}

In the iterative channel equalization algorithm with soft decision feedback detection, the equalizer is applied on each data block $\mathbf{Y}$ iteratively by exploiting the feedback of the extrinsic information of equalized bits, which is fed back to the equalizer as the a-priori information for the next iteration. The equalizer uses this information to compute a soft estimation of transmitted symbols and equalizes the received symbols in the FD based on the MMSE criterion. After equalization, a (log-likelihood ratio) LLR calculator uses the output and the coefficients of the equalizer to compute the extrinsic information of the equalized bits.

The block diagram of the IFDE algorithm applied in our experiment is illustrated in Figure 5. Synchronization and Doppler compensation are performed to obtain $\mathbf{y}$, and CSbased channel estimation algorithm is used to obtain the estimated CIRs $\hat{\mathbf{h}}$.

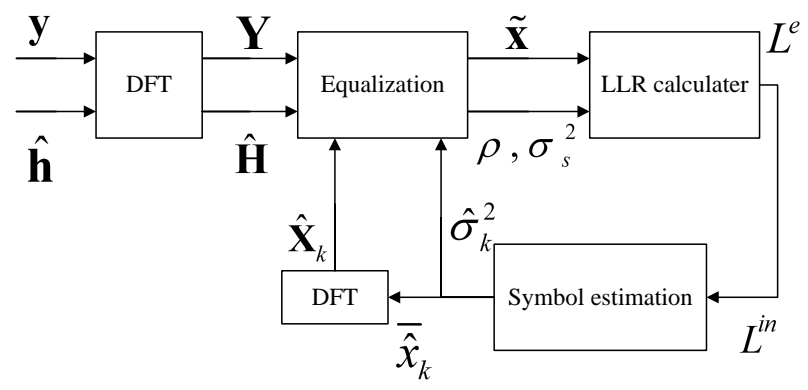

Fig. 5. Block diagram of the IFDE algorithm

\section{A. Symbol Estimation}

The symbol estimation module in the equalizer uses the apriori information $L^{i n}(b(t))$ to obtain a soft estimation $\hat{\mathbf{x}}$ of the transmitted symbols, including their mean $\overline{\hat{x}}_{k}$ and variance $\hat{\sigma}_{k}^{2}$. The a-priori information LLR and the a-posteriori information LLR of the information bits are defined as

$$
\begin{aligned}
L^{\text {in }}(b(t)) & =\ln \frac{\operatorname{Pr}(b(t)=1)}{\operatorname{Pr}(b(t)=0)} \\
L^{\text {out }}(b(t) \mid \tilde{x}(k)) & =\ln \frac{\operatorname{Pr}(b(t)=1 \mid \tilde{x}(k))}{\operatorname{Pr}(b(t)=0 \mid \tilde{x}(k))}
\end{aligned}
$$

where $t=(k-1) M+i, i=1,2, \ldots, M, \tilde{x}(k)$ is the $k$-th symbol after equalization, and $\operatorname{Pr}(\cdot)$ stands for the probability operator.

Let us introduce $\mathbf{b}_{m}=\left[b_{m, 1}, b_{m, 2}, \ldots, b_{m, M}\right]^{T}$ as the vector of information bits that are mapped to the symbol $s_{m}$, where $b_{m, i} \in\{0,1\}, i=1,2, \ldots, M$. We can calculate $\overline{\hat{x}}_{k}$ and $\hat{\sigma}_{k}^{2}$ as

$$
\begin{gathered}
\overline{\hat{x}}_{k}=\sum_{s_{m} \in S} s_{m} \operatorname{Pr}\left(\hat{x}_{k}=s_{m}\right) \\
=\sum_{s_{m} \in S} s_{m} \prod_{t=M(k-1)+1}^{M k} \operatorname{Pr}\left(b(t)=b_{m, i}\right) \\
\hat{\sigma}_{k}^{2}=\sum_{s_{m} \in S}\left|s_{m}\right|^{2} \operatorname{Pr}\left(\hat{x}_{k}=s_{m}\right)-\left|\hat{\hat{x}}_{k}\right|^{2} .
\end{gathered}
$$

The bit probabilities in (7) and (8) are determined by the a-priori information $L^{i n}(b(t))$ as

$$
\operatorname{Pr}\left(b(t)=b_{m, i}\right)=\frac{\left(1-b_{m, i}\right) P_{0}+b_{m, i} P_{1}}{P_{0}+P_{1}}
$$

where $P_{0}=\exp \left(-L^{i n}(b(t)) / 2\right)$ and $P_{1}=$ $\exp \left(L^{i n}(b(t)) / 2\right)$.

\section{B. Frequency-Domain Equalization}

The soft estimation of the transmitted symbols are first converted into the FD by a DFT module as

$$
\hat{\mathbf{X}}_{k}=\mathbf{F}\left[\overline{\hat{x}}_{1}, \ldots, \overline{\hat{x}}_{k-1}, 0, \overline{\hat{x}}_{k+1}, \ldots, \overline{\hat{x}}_{N}\right]^{T} .
$$

Based on the MMSE criterion, the equalizer processes the symbols in the frequency domain. The equalized symbols can be represented in the frequency domain as

$$
\tilde{\mathbf{X}}(k)=\mathbf{W Y}-\mathbf{Q} \hat{\mathbf{X}}_{k}
$$


where the equalizer coefficients $\mathbf{W}$ and $\mathbf{Q}$ are given by

$$
\begin{gathered}
\mathbf{G}=\hat{\mathbf{H}}^{H}\left(\overline{\hat{\sigma}}^{2} \hat{\mathbf{H}} \hat{\mathbf{H}}^{H}+\sigma_{w}^{2} \mathbf{I}_{N}\right)^{-1} \\
\mathbf{W}=\frac{1}{1+\left(1-\overline{\hat{\sigma}}^{2}\right) \operatorname{Tr}\{\mathbf{G} \hat{\mathbf{H}}\} / N} \mathbf{G} \\
\mathbf{Q}=\mathbf{W} \hat{\mathbf{H}} .
\end{gathered}
$$

Here $\hat{\mathbf{H}}$ is the estimation of the frequency-domain channel matrix, $\overline{\hat{\sigma}}^{2}=\sum_{k=1}^{N} \hat{\sigma}_{k}^{2} / N$ is the mean of the variance of the soft estimated symbols, and $(\cdot)^{-1}$ and $\operatorname{Tr}\{\cdot\}$ denote matrix inversion and matrix trace, respectively.

\section{Analysis of the Output of Equalizer}

After equalization, the TD symbols are presented as

$$
\tilde{\mathbf{x}}=\operatorname{diag}\left\{\mathbf{F}^{H} \tilde{\mathbf{X}}\right\}
$$

where $\tilde{\mathbf{X}}$ is an $N \times N$ matrix whose $k$-th column is $\tilde{\mathbf{X}}(k)$ in (11), and $\operatorname{diag}\{\cdot\}$ denotes a column vector composed by the diagonal elements of a matrix. Since the equalized symbols are commonly assumed to be approximately Gaussian distributed for given transmitted symbols $\mathbf{x}$, the TD symbols can be approximately expressed as

$$
\tilde{\mathbf{x}}=\rho \mathbf{x}+\omega
$$

where $\rho$ is an $N \times N$ diagonal matrix with $\rho(k)$ as its $k$-th diagonal element, and $\omega$ is a column vector of white Gaussian noise with zero mean and variance $\sigma_{s}^{2}$. They are calculated by

$$
\begin{gathered}
\rho=\operatorname{diag}\left\{\mathbf{F}^{H} \mathbf{Q F}\right\} \\
\sigma_{s}^{2}=\frac{\sum_{k=1}^{N}\left(|\rho(k)|-\overline{\hat{\sigma}}^{2}|\rho(k)|^{2}\right)}{N+\left(1-\overline{\hat{\sigma}}^{2}\right) \operatorname{Tr}\{\mathbf{G} \hat{\mathbf{H}}\}} .
\end{gathered}
$$

Using (17) and (18), the conditional probability density function of $\tilde{x}(k)$ can be written as

$$
\operatorname{Pr}\left(\tilde{x}(k) \mid s_{m}\right)=\frac{1}{\pi \sigma_{s}^{2}} \exp \left(-\frac{\left|\tilde{x}(k)-\rho(k) s_{m}\right|^{2}}{\sigma_{s}^{2}}\right) .
$$

\section{Extrinsic Information Computation and Iteration}

The equalized symbols $\tilde{x}(k)$ and the parameters $\rho(k), \sigma_{s}^{2}$ are used to compute the extrinsic information of the equalized bits which will be used for the next iteration. The extrinsic information is obtained by subtracting the a-priori information from the a-posteriori information. Using (5) and (6), the extrinsic information LLR after equalization is given by

$$
\begin{aligned}
& L^{e}(b(t))=L^{\text {out }}(b(t) \mid \tilde{x}(k))-L^{\text {in }}(b(t)) \\
& =\ln \frac{\sum_{\forall s_{m}: b_{m, i}=1} \operatorname{Pr}\left(\tilde{x}(k) \mid s_{m}\right) \prod_{\forall j: j \neq i} \operatorname{Pr}\left(b((k-1) M+j)=b_{m, j}\right)}{\sum_{\forall s_{m}: b_{m, i}=0} \operatorname{Pr}\left(\tilde{x}(k) \mid s_{m}\right) \prod_{\forall j: j \neq i} \operatorname{Pr}\left(b((k-1) M+j)=b_{m, j}\right)}
\end{aligned}
$$

where the a-priori probability can be calculated using (9), and the conditional probability density function is given by (19). The extrinsic information of the equalized bits $L^{e}(b(t))$ obtained from the LLR calculator is fed back to the equalizer

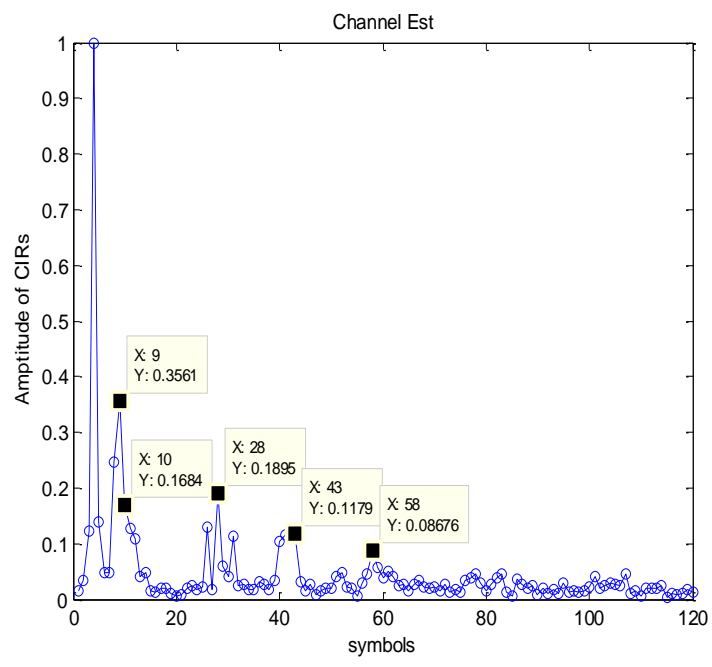

Fig. 6. The estimated CIRs

as the a-priori information for the next iteration. The correlation between the a-priori information and the a-posteriori information increases with the number of iterations. Thus, the improvement of the IFDE algorithm diminishes gradually with the number of iterations. After several iterations, the equalizer makes a hard decision based on the extrinsic information to obtain the transmitted bits.

\section{EXPERIMENT RESULTS}

In this section, we present the results obtained by applying the SC-IFDE algorithm to process the data received during our UA communication experiment conducted in the Indian Ocean off Rottnest Island, Western Australia, in December 2012. In the experiment, there was one transmitting transducer and one receiving hydrophone. The distance between the transmitter and receiver varied from $125 \mathrm{~m}$ to $10 \mathrm{~km}$. In this paper, we focus on the distances of $1 \mathrm{~km}$ and $2 \mathrm{~km}$.

The information bit rate of the experimental system is 8 kbps. The information bits are mapped into QPSK symbols and the duration of each block is about 0.5 second (4000 information bits). The ocean surface was rough during the experiment. Sometimes the change of the UA channel during one block is so large that some symbols may not be detected successfully and some symbols may have a very low signal-tonoise ratio (SNR). In the following results, all data processed is synchronized successfully, and the SNR is larger than OdB.

Figure 6 shows the system's equivalent normalized CIRs over $1 \mathrm{~km}$ range estimated by the channel estimation module. It can be seen from Figure 6 that the multipath span of the UA channel is close to 100 symbols (about $25 \mathrm{~ms}$ ) and there are multiple peaks in the CIR profile. The SNR estimated by using the training sequence is around $15 \mathrm{~dB}$.

We randomly choose one data block received by the hydrophone to present the scatter plots of the received symbols and the equalized symbols in Figure 7. The scatter plots show 


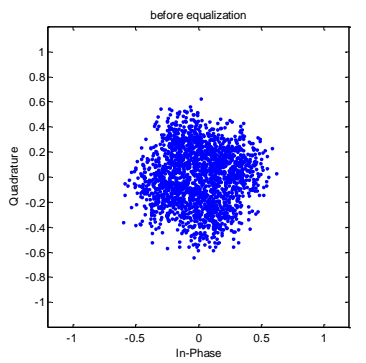

(a) before equalization

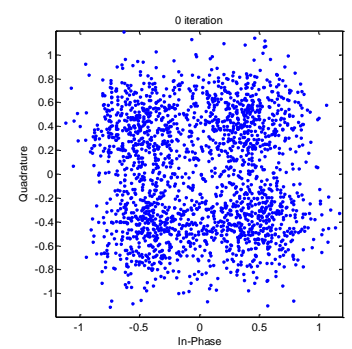

(b) linear MMSE equalization

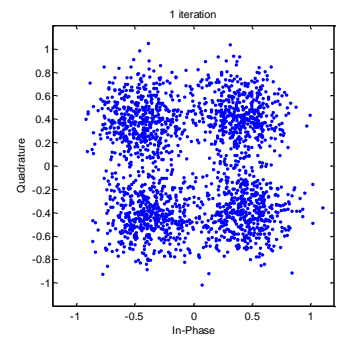

(c) after one iteration

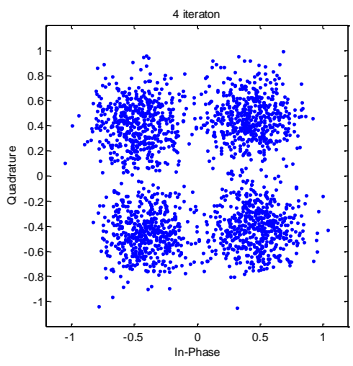

(d) after four iterations

Fig. 7. Scatter plots of the system over $1 \mathrm{~km}$ range with QPSK constellations.

TABLE I

THE BER PERFORMANCE OF THE SC-IFDE AND THE LINEAR MMSE EQUALIZATION ALGORITHMS

\begin{tabular}{|c|c|c|c|c|c|}
\hline \multirow{2}{*}{ Distance } & Linear MMSE & \multicolumn{4}{|c|}{ SC-IFDE } \\
\cline { 2 - 6 } & 0 iteration & 1 iteration & 2 iterations & 3 iterations & 4 iterations \\
\hline $1 \mathrm{~km}$ & $5.58 \%$ & $3.81 \%$ & $3.49 \%$ & $3.33 \%$ & $3.21 \%$ \\
\hline $2 \mathrm{~km}$ & $14.48 \%$ & $10.35 \%$ & $9.89 \%$ & $9.74 \%$ & $9.66 \%$ \\
\hline
\end{tabular}

that most of the QPSK symbols can be properly aggregated into the normalized modulation constellations after equalization, and the equalized symbols produced by the SC-IFDE algorith$\mathrm{m}$ are gathered more compactly to the normalized modulation constellations after several iterations. It can be clearly seen from Figure 7 that the gathering of the scatter plot after four iterations is better than that without iteration. In fact, the SCIFDE without iteration is the linear MMSE equalization. It can also be observed from Figure 7 that the improvement from the first iteration to the fourth iteration is not obvious. This is due to the fact that the correlation between the a-priori information and the a-posteriori information increases with iterations.

Table I shows the uncoded BER performance of the SCIFDE algorithm averaged over 35 data blocks (including about $10^{5}$ information bits). In fact, the SC-IFDE without iteration is the linear MMSE equalization. As shown in Table I, in the 1 $\mathrm{km}$ range, the average BER of the linear MMSE equalization is $5.58 \%$. With the increasing number of iterations, the average BER yielded by the SC-IFDE algorithm reduces. After four iterations, the SC-IFDE algorithm achieves an average BER of $3.21 \%$, which is almost $3 \mathrm{~dB}$ lower than that of the linear MMSE equalization. Table I also shows the average system BER over a $2 \mathrm{~km}$ range, where the average BER is reduced from $14.48 \%$ to $9.66 \%$ after four iterations. Therefore, in the UA communication signal processing, the SC-IFDE algorithm can significantly improve the BER performance compared with the linear MMSE equalization.

We would like to mention that we have used the singlecarrier Turbo frequency-domain equalization (SC-TFDE) to process the data collected in our experiment. Different from the SC-IFDE without using decoder, the SC-TFDE works with the exchange of the extrinsic information between the equal- izer and decoder. Because of the decoder's error correction capability, the SC-TFDE can achieve much lower BERs than that of the SC-IFDE. In the $1 \mathrm{~km}$ SISO system, the information bits error number of the SC-TFDE is zero. However, the system information rate is reduced in order to apply the SCTFDE. Such BER-rate tradeoff is useful for practical UA communication systems.

\section{CONCLUSION}

In this paper, we applied the SC-IFDE algorithm to process the data received during our UA communication experiment conducted in December 2012 in the Indian Ocean Rottnest Island, Western Australia. Different to the linear MMSE equalization, the SC-IFDE processes the received symbols iteratively by exploiting the feedback of the extrinsic information which greatly improves the equalizer performance. The BER performance results show that the SC-IFDE algorithm outperforms the linear MMSE equalization in UA communication. On the other hand, the information rate of the SC-IFDE is higher than the SC-TFDE using the same symbol block structure.

\section{REFERENCES}

[1] M. Stojanovic and J. Preisig, "Underwater acoustic communication channels: Propagation models and statistical characterization," IEEE Commun. Mag.,vol. 47, pp. 84-89, Jan. 2009.

[2] A. Baggeroer, "An overview of acoustic communications from 2000 - 2012," Proc. Underwater Communications: Channel Modelling \& Validation, Sestri Levante, Italy, Sep. 12-14, 2012.

[3] J. Zhang and Y. R. Zheng, "Frequency-domain Turbo equalization with soft successive interference cancellation for single carrier MIMO underwater acoustic communications," IEEE Trans. Wireless Commun.,vol. 10, pp. 2872-2882, Sep. 2011.

[4] F. Pancaldi, G. M. Vitetta, R. Kalbasi, N. Al-Dhahir, M. Uysal, and H. Mheidat, "Single-carrier frequency domain equalization," IEEE Signal Process. Mag.,vol. 25, pp. 37-56, Sep. 2008.

[5] N. Benvenuto and S. Tomasin, "On the comparison between OFDM and single carrier modulation with a DFE using a frequency-domain feedforward filter," IEEE Trans. Commun.,vol. 50, pp. 947-955, June 2002.

[6] N. Benvenuto and S. Tomasin, "Iterative design and detection of a DFE in the frequency domain," IEEE Trans. Commun.,vol. 53, pp. 1867-1875, Nov. 2005.

[7] B. $\mathrm{Ng}$, C. Lam, and D. Falconer, "Turbo frequency domain equalization for single-carrier broadband wireless systems," IEEE Trans. Wireless Commun.,vol. 6, pp. 759-767, Feb. 2007. 